\title{
ITA-CRAO MINOR PLANET SURVEY: RESULTS AND PROSPECTS
}

\author{
N.S. CHERNYKH \\ Crimean Astrophysical Observatory \\ Nauchnyj, Crimea, Ukraine
}

AND

A. G. SOKOLSKY

Institute of Theoretical Astronomy, Russian Acad. of Sciences St. Petersburg, Russia

\begin{abstract}
Results of minor planet observation programme that is made jointly by the Institute of Theoretical Astronomy and the Crimean Astrophysical Observatory during three decades with the Zeiss $40-\mathrm{cm}$ double astrograph at Crimea are presented. Almost all permanently numbered minor planets were observed. A catalogue of astrometrical results obtained in the course of this survey contains more than 60 thousand minor planet positions. Many thousand unnumbered asteroids were discovered, 875 of which had received permanent numbers by June of 1996.

In the development of this survey programme we plan to use another Crimean telescope - the fast 64-cm telescope of Richter and Slevogt system. We intend to equip it with a CCD camera and use it for searching and observation of the Near Earth Asteroids. In the future this CCD-telescope must be a part of the national Russian network for Near Earth Asteroid (NEA) observations.
\end{abstract}

\section{Introduction}

The regular photographic survey of minor planets with the $40-\mathrm{cm}$ double astrograph was begun at the Crimean Astrophysical Observatory (CrAO) in 1963, ten years after the Simeiz minor planet programme had been stopped. Restoration of this programme was an important event, since from the mid-1950s none of the Soviet observatories was concerned with minor planet observations. A new Crimean minor planet programme was started according to the initiative of the Leningrad Institute of Theoretical Astronomy (ITA), as a result of collaboration with the Crimean Astrophysical Observatory. Both institutions had supplied the observing team with everything it needs for the work, including a telescope, a star plate comparator,

I. M. Wytrzyszczak, J. H. Lieske and R. A. Feldman (eds.),

Dynamics and Astrometry of Natural and Artificial Celestial Bodies, 567, 1997.

(C) 1997 Kluwer Academic Publishers. Printed in the Netherlands. 


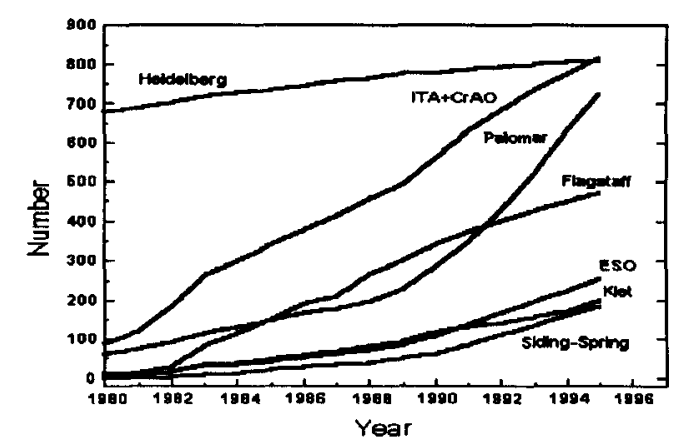

Figure 1. Number of minor planets discovered at different observatories.

two Ascorecord co-ordinatometers, two computers, necessary catalogues, ephemerides and computer programmes.

\section{Results}

The observations are carried out with a $40-\mathrm{cm} \mathrm{f} / 4$ double astrograph. It was built by Carl Zeiss Jena firm during 1939-1944 and was installed at the Observatory at Nauchnyj in 1948, in the first stage of building of CrAO. The astrograph gives a field of $10^{\circ} \times 10^{\circ}$ on plates of $30 \times 30 \mathrm{~cm}$. A 90-min exposure, which is maximum for $\mathrm{f} / 4$, gives on the ORWO $\mathrm{ZU} 21$ plates the limiting magnitude of 18 (in system B). In good conditions a plate records from 30 to 50 asteroids, of which a great part are not numbered.

In the past years about 100-120 pairs plates were taken annually covering 5000-7000 square degrees in the ecliptical zone. At the average, $500-600$ numbered minor planets were observed during a year and over 500-600 unnumbered asteroids were discovered. As a result, it yielded from 2000 to 3000 positions in a year. Altogether, during three decades about $90 \%$ of known numbered minor planets have been observed, more than 16000 unnumbered asteroids have been detected, and more than 60000 astrometric positions have been measured. Moreover, 1200 positions of 60 observed comets were determined and two new short-period comets were discovered. Some "old" minor planets having uncertain orbits were rediscovered and observed in the course of survey and orbits of many minor planets were improved in ITA using the Crimean observations.

From among new minor planets discovered with the Crimean astrograph, 875 have obtained permanent numbers by June of 1996. For many years our results in the discovery of minor planets were a part of the record of the International Service of minor planets (Marsden, 1996). Figure 1 shows the data of our discovery of minor planets numbered within several last years, together with the results of the other most outstanding surveys.

It may not be out of place to mention here that some minor planets discovered at $\mathrm{CrAO}$ had received Polish names: 
- 2163 Korczak

- 2519 Annagerman

- 3836 Lem

- 5889 Mickiewicz

In the last years the observational activity of the Crimean asteroid programme decreased owing to the known difficulties experienced by Russia and the other countries of the former Soviet Union. Nevertheless, some new observational results have been obtained, including the astrometric observations of 4179 Toutatis, 1620 Geographos and $1993 \mathrm{MF}$ - and the works on the linking the Ascorecord with the personal computer and working up some new computing programmes for the reduction computations and processing of the observations.

\section{New ideas and plans}

In 1991 the ITA initiated a detailed programme of NEAs research in Russia. For the co-ordination of this work, the International Institute for Problems of Asteroid Hazards (IIPAH), closely connected with the ITA, was created. It became the center that leads the investigations on the Near-Earth objects (NEOs) and cosmic impact hazard problems not only in Russia, but in the other countries of the former SU as well ("Asteroid hazard" Conference, 1992).

One of the most important components of this programme is the organization of a Russian network for the discovery and following up of the Near-Earth asteroids and comets. As is well known, ordinary minor planet surveys cannot ensure the discovery of a great amount of NEOs, and the special programmes, instruments and methods are necessary for this aim.

The Crimean 40-cm f/4 astrograph is not suitable for the observation of such fast-moving objects like NEOs, but now we have a telescope more appropriate for our purpose. It is fast, $64-\mathrm{cm}, \mathrm{f} / 1.4$ Richter-Slevogt camera.

We already obtained several dozen plates with it for the positions of asteroid 6489 (1991 JX) during its approach to the Earth in June of 1995 and some other objects.

The optical system of Richter and Slevogt is rather rare. Like a Schmidt it has a spherical main mirror but its corrector is more compound and it consists of two lenses, the first of which is concavo-convex and the other is convexo-plane. One of the peculiarities of this optical system is the short length of it: the distance from correcting lenses to main mirror doesn't exceed the diameter. The corrected field of view of the Crimean $64-\mathrm{cm}$ camera is $6 \mathrm{~cm}$ in diameter, which equals only 4 degrees. Obviously, it is insufficiently for the making of photographical survey but is quite enough for the following up of individual objects. 
TABLE 1. Number of predicted NEAs and main belt asteroids.

\begin{tabular}{lcccccc}
\hline Limiting magnitude V & 15 & 16 & 17 & 18 & 19 & 20 \\
\hline Number of NEAs & 0.2 & 0.6 & 1.5 & 3.7 & 9.3 & 23.4 \\
Number of asteroids & 600 & 1300 & 3100 & 7500 & 18000 & 43000 \\
\hline
\end{tabular}

We plan to use this telescope as an astrograph only in the first stage of its work. The next step will be equipping it with the CCD camera. The limiting magnitude for the 64-cm telescope with the CCD is expected to be about $\mathrm{V}=20$. Now we have a CCD matrix of Russian manufacture with $1040 \times 1160$ pixels of 16 micron size. It will give a field of $64^{\prime} \times 72^{\prime}$ in the focal plane of our telescope. In the case of observation in scanning at the sidereal rate, it will give the coverage about 50 square degrees per night and as much as 6000 square degrees during a year. The probability of discovery of NEAs on such an area of the sky may be estimated on the basis of the results of paper by E. Helin and R. Dunbar (1990). Table 1 shows the possible number of NEAs, and the amount of main belt asteroids in the same sky area [estimated with van Houten et al. (1970) statistics] in funtion of asteroid's limiting magnitude.

We hope to have a CCD camera installed at the telescope in the not too distant future. The Crimean 64-cm telescope, after equipping it with the CCD camera, will play a role of the experimental sample. The experience that will be gained in the course of work with it will be useful for the development of the national Russian network for CCD asteroid survey. Some observatories of the other countries from the former SU are interested in the participation in this programme, which will be a part of the Spaceguard Survey. The extended Russian NEO-network may consist of about two dozen observatories that have telescopes of $\mathbf{4 0}$ or more centimeters in diameter. Being equipped with a CCD, these telescopes could record the NEOs of magnitude 19 and fainter.

The Crimean old $40-\mathrm{cm}$ double astrograph is still an effective device for the discovery and astrometry of ordinary minor planets. It has not yet reached the limit of its resources and will be used, as before, for the survey of main-belt asteroids.

\section{References}

Proceedings of the All-Union Conference "Asteroid Hazard": 1992, St. Petersburg, Russia (in Russian).

Helin, E.F., Dunbar, R.S.: 1990, "Search techniques for Near-Earth Asteroids", Vistas Astron. 33, part 1.

van Houten, C.J., van Houten-Groeneveld, I., Herget, P., Gehrels, T.: 1970, "The Palomar-Leiden survey of faint minor planets", Astron. Astrophys., Suppl. Ser. 2,No.5.

Marsden, B.G.: 1996, "From telescope to MPC: Organizing the minor planets", in: Dynamics, Ephemerides and Astrometry of the Solar System, IAU Symposium 172 (S. Ferraz-Mello, B. Morando, J.-E. Arlot, eds), Kluwer, Dordrecht. 